\title{
Randomized Controlled Clinical Trial
}

National Cancer Institute

\section{Source}

National Cancer Institute. Randomized Controlled Clinical Trial. NCI Thesaurus. Code C46079.

A study in which the participants are assigned by chance to separate groups to evaluate treatments. The study also includes a control group, which is undergoing the same rigors of the study with placebo treatment or standard treatment. The trial can also be controlled by the addition of a test group to ensure there are no factors present to skew the results and by the inclusion of peer-review analysis by appropriate government officials. 2005 